\title{
SUMOylation of DISC1: A Potential Role in Neural Progenitor Proliferation in the Developing Cortex
}

\author{
Stephanie Tankou ${ }^{a}$ Kazuhiro Ishii ${ }^{a}$ Christina Elliott $^{\mathrm{e}}$ Krishna C. Yalla \\ Jon P. Day ${ }^{\mathrm{e}}$ Keiko Furukori ${ }^{\mathrm{a}}$ Ken-ichiro Kubo ${ }^{\mathrm{g}}$ Nicholas J. Brandon $^{\mathrm{c}}$ \\ Qiyi Tang $^{d}$ Gary Hayward $^{b}$ Kazunori Nakajimag Miles D. Houslay ${ }^{f}$ \\ Atsushi Kamiya $^{a}$ George S. Baillie ${ }^{e}$ Koko Ishizuka ${ }^{a}$ Akira Sawa $^{a}$
}

Departments of a Psychiatry and ${ }^{b}$ Pharmacology, The Johns Hopkins University, Baltimore, Md., ' AstraZeneca Neuroscience iMED, Cambridge, Mass., and d Department of Microbiology, Howard University College of

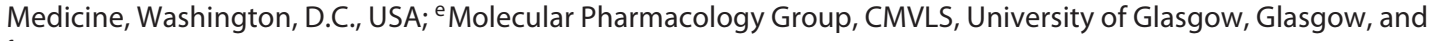
${ }^{f}$ Institute of Pharmaceutical Sciences, King's College London, London, UK; ${ }^{9}$ Department of Anatomy, Keio University School of Medicine, Tokyo, Japan

\section{Key Words}

DISC1 · SUMOylation · Posttranslational modification ·

Cortical development · Mental illness

\begin{abstract}
DISC1 is a multifunctional, intracellular scaffold protein. At the cellular level, DISC1 plays a pivotal role in neural progenitor proliferation, migration, and synaptic maturation. Perturbation of the biological pathways involving DISC1 is known to lead to behavioral changes in rodents, which supports a clinical report of a Scottish pedigree in which the majority of family members with disruption of the DISC1 gene manifest depression, schizophrenia, and related mental conditions. The discrepancy between modest evidence in genetics and strong biological support for the role of DISC1 in mental conditions suggests a working hypothesis that regulation of DISC1 at the protein level, such as posttranslational modification, may play a role in the pathology
\end{abstract}

of mental conditions. In this study, we report on the SUMOylation of DISC1. This posttranslational modification occurs on lysine residues where the small ubiquitin-related modifier (SUMO) and its homologs are conjugated to a large number of cellular proteins, which in turn regulates their subcellular distribution and protein stability. By using in silico, biochemical, and cell-biological approaches, we now demonstrate that human DISC1 is SUMOylated at one specific lysine 643 (K643). We also show that this residue is crucial for proper neural progenitor proliferation in the developing cortex.

(c) 2016 S. Karger AG, Basel

\section{Introduction}

Over the past several decades, we have used genetic and neuropathological approaches to explore compelling molecular leads to address the mechanisms of neurologi-

\section{KARGER}

E-Mail karger@karger.com

www.karger.com/mnp 
cal and neuropsychiatric disorders [1-3]. For example, tau and $\alpha$-synuclein proteins have been underscored as major components of inclusion bodies that occur in the brains of patients with Alzheimer's disease and Parkinson's disease, respectively $[4,5]$. The tau and $\alpha$-synuclein proteins are pathologically phosphorylated $[6,7]$, and the pathological roles of these posttranslational modifications have been elucidated [8]. Likewise, pathological implications of other posttranslational modifications have also been reported [9].

The DISC1 locus has received broad attention in the context of psychiatric illness, since a balanced translocation that segregated in a Scottish pedigree with schizophrenia and depression was reported [10]. Since the discovery of the gene, many neurobiologists have studied the biological function of DISC1. At the cellular level, DISC1 plays a pivotal role in neural progenitor proliferation, migration, and synaptic maturation [11, 12]. Perturbation of the biological pathways involving DISC1 is known to lead to behavioral changes in rodents [1321]. In contrast to biological results that support the role for DISC1 in mental conditions, genetic evidence to support the contribution of the DISC1 gene in sporadic schizophrenia has been modest [22]. The discrepancy between modest evidence in genetics and strong biological support for the role of DISC1 in mental conditions led us to propose a working hypothesis that regulation of DISC1 at the protein level, such as posttranslational modification, may play a role in its function in pathology.

DISC1 is located in multiple subcellular domains, including the centrosome, postsynaptic density, and the nucleus $[11,23,24]$. Like tau and $\alpha$-synuclein, specific phosphorylation on DISC1 affects its localization to the centrosome and plays a crucial role in neurodevelopment [25]. Protein targeting to the nucleus is also influenced by posttranslational modifications, such as SUMOylation [26]. SUMOylation occurs on lysine residues whereby small ubiquitin-related modifiers (SUMO) and homologs are conjugated to proteins [27]. SUMOylation has emerged as a major regulator of nuclear function, including DNA replication, DNA damage response, and transcription [28-30]. Furthermore, an important role for SUMOylation in protein trafficking to the nucleus has also been reported [31].

In the present study, we show that human DISC1 is SUMOylated at lysine 643 (K643) with in silico, biochemical, and cell-biological approaches. This specific SUMOylation site of DISC1 regulates neural progenitor proliferation in the developing cortex.

\section{Methods}

Animals

Pregnant C57BL/6 mice were purchased from Charles River.

\section{Constructs and Antibodies}

A K643A variant of human DISC1 or a K640A variant of mouse DISC1 (mDISC1) was introduced by PCR-based mutagenesis as mentioned previously [25]. All constructs in the present study were created as described previously [24,32]. We used a short hairpin RNA (shRNA) construct of DISC1 that was published previously (the targeting sequence is $5^{\prime}$-GGCAAACACTGTGAAGTGC-3') [24]. We used a control RNAi construct with a scrambled sequence without homology to any known messenger RNA [33]. GFP-tagged SUMO1, FLAG-tagged SUMO1, and Myc-tagged Ubc9 constructs were generous gifts from Dr. Pandolfi (Beth Israel Deaconess Cancer Center, Boston, Mass., USA), Dr. Hayward (The Johns Hopkins University, Baltimore, Md., USA), and Dr. Matunis (Johns Hopkins Bloomberg School of Public Health, Baltimore, Md., USA), respectively. The following antibodies were used: mouse monoclonal anti-HA antibody (Covance); rabbit polyclonal anti-FLAG antibody (Sigma); rabbit polyclonal antiSUMO1 antibody (Zymed); mouse monoclonal anti-BrdU antibody (BD Biosciences); anti-DISC1-C2 antibody [34], and a rabbit polyclonal anti-human DISC1 antibody (kindly provided by Dr. Akiyama, University of Tokyo, Tokyo, Japan) [35-37].

In silico Prediction of SUMOylation Site(s) in DISC1

Prediction of DISC1 SUMOylation sites was made by using the SUMOplot ${ }^{\mathrm{TM}}$ Analysis Program from Abgent available at http:// www.abgent.com/sumoplot. The score indicates the probability that the targeted lysine is SUMOylated.

\section{Recombinant Proteins}

To obtain C-terminal DISC1-fragmented glutathione S-transferase (GST-DISC1-C) fusion proteins, the C-terminal of DISC1 (amino acids 598-854) was inserted into the pGEX vector. The expression plasmids were introduced into BL21-competent Escherichia coli with $0.1 \mathrm{mM}$ IPTG. Recombinant proteins were purified from E. coli with glutathione Sepharose or amylase beads.

\section{In vitro SUMOylation Assay}

In vitro SUMOylation was assayed by use of the SUMOylation assay kit (Boston Biochem). Briefly, reactions contained $10 \mu \mathrm{g}$ GST-DISC1-C, $50 \mu \mathrm{M}$ SUMO proteins, $100 \mathrm{nM}$ SUMO-activating enzyme E1, $5 \mu \mathrm{M} \mathrm{Ubc9}$, and $1 \mathrm{mM} \mathrm{Mg-ATP}$ in buffer $(50 \mathrm{~mm}$ HEPES, $\mathrm{pH} 8.0,100 \mathrm{mM} \mathrm{NaCl}, 1 \mathrm{~mm}$ DTT), and were incubated at $37^{\circ} \mathrm{C}$ for $2 \mathrm{~h}$. The reactions were also run in the absence of ATP as a negative control. Proteins were separated on a NuPAGE $4-12 \%$ Bis-Tris gel (Invitrogen) and visualized with SimplyBlue SafeStain (Invitrogen). For Western blotting, an 8\% Tris-Glycine gel (Invitrogen) was used.

\section{Ubc9 Fusion-Directed SUMOylation Assay}

A Ubc9 Fusion-Directed SUMOylation (UFDS) assay was performed as previously described [32], with minor modifications. Briefly, HEK293 cells were transfected with the construct of DISC1Ubc9 or DISC1-Ubc9 (C93S) together with the SUMO1-GFP construct, and lysed $48 \mathrm{~h}$ after transfection. Immunoblots were conducted with the anti-DISC1 antibody described above [35]. 
Fig. 1. SUMOylation of DISC1-C at lysine residue 643 (K643) in vitro. a DISC1 SUMOylation sites predicted by the use of the Abgent SUMOplot. b In vitro SUMOylation assay with GST-DISC1-C. Proteins were separated on a $4-12 \%$ BisTris gradient gel. Coomassie blue staining showed that DISC1-C was covalently conjugated to SUMO1, SUMO2, and SUMO3. Red arrowheads indicate SUMOylated forms of DISC1-C. c In vitro SUMOylation assay with GST-DISC1-C or mutant DISC1-C where K643 was substituted by alanine (DISC1-C-K643A). 8\% Tris-Glycine gel electrophoresis and immunoblotting showed that DISC1-C but not DISC1C-K643A was SUMOylated. Red arrowheads indicate SUMOylated forms of DISC1-C. Colors refer to the online version only. IB = Immunoblot.

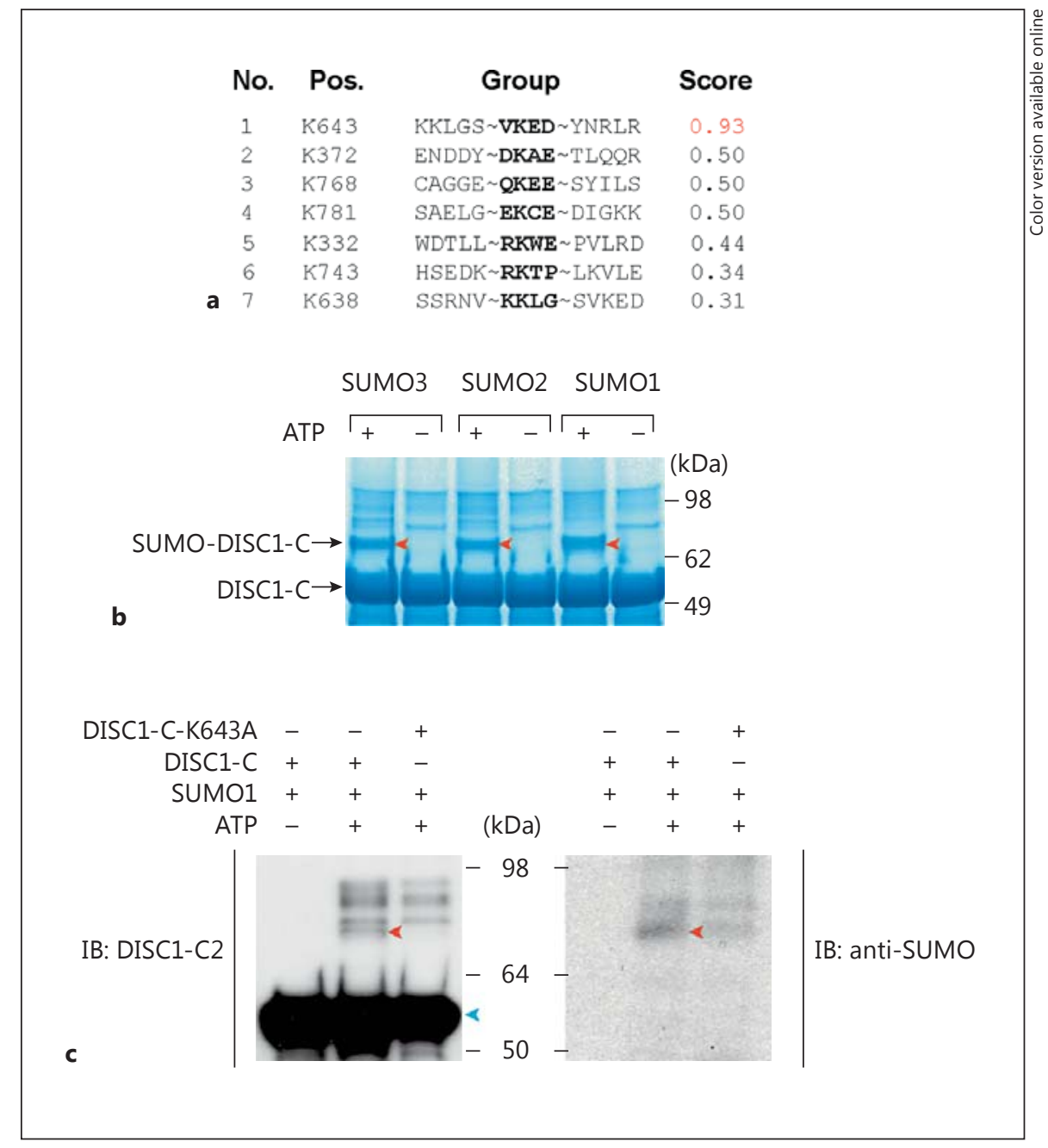

\section{Cell Culture}

COS7 cells were used to assay SUMOylation on DISC1. Cells were grown in Dulbecco's modified Eagle's medium with 10\% FBS. The expression constructs were transfected into cells by using FuGENE ${ }^{\circledR} 6$ (Roche Applied Sciences). U373 cells were transfected with a DISC1 or DISC1-K643A construct to examine the nuclear translocation of SUMOylated DISC1. Cells were immunostained as previously described [25]. The intensity of immunostaining of DISC1 in the nucleus versus the whole cell was quantified with Metamorph (Molecular Devices) for all experimental groups. The intensity ratio of the signal for at least 30 cells per group was analyzed in three independent experiments.

\section{Immunoprecipitation}

Cells were lysed in a RIPA buffer (50 mM HEPES, pH 7.4, 150 $\mathrm{mm} \mathrm{NaCl}, 5 \mathrm{~mm} \mathrm{MgCl}_{2}, 5 \mathrm{~mm}$ DTT, $1 \mathrm{~mm}$ PMSF, $1 \mathrm{~mm}$ EDTA, $1 \%$ Triton X-100) containing protease inhibitor mixture (Roche Applied Sciences). Supernatant fractions were obtained from lysates after centrifugation at 13,000 rpm for $15 \mathrm{~min}$. Soluble fractions were used for immunoprecipitation as described previously [25].

\section{In utero Electroporation}

Pregnant C57BL/6 mice at embryonic (E) day 13 were deeply anesthetized by intraperitoneal administration of 2,2,2-tribromoethanol in tert-amyl alcohol $(0.4 \mathrm{mg} / \mathrm{g})$, and intrauterine embryos were surgically manipulated as described previously [24, 38]. Plasmid solutions in volumes of $1-2 \mu \mathrm{l}$ containing RNAi plasmids $(2 \mu \mathrm{g} / \mu \mathrm{l})$ together with CAG-driven mDISC1 with mutations in the RNAi targeting site expression vector $(1 \mu \mathrm{g} / \mu \mathrm{l})$ and/or CAGdriven GFP expression vector $(1 \mu \mathrm{g} / \mu \mathrm{l})$ were injected into the lateral ventricles. To use the same amount of DNA for each condition, an empty expression vector (pCAGGS1) was used as necessary. Electronic pulses (35 V, $50 \mathrm{~ms}, 4$ times) were applied using an electroporator (CUY21E, Tokiwa Science) with a forceps-type electrode (CUY650-5, Tokiwa Science).

\section{BrdU Incorporation Assay and Brain Slice Preparation}

Incorporation of BrdU was performed as previously described [25], with minor modifications. $50 \mathrm{mg} / \mathrm{kg}$ BrdU (Sigma) was injected intraperitoneally into E13 pregnant mice $48 \mathrm{~h}$ after electroporation. Then, $2 \mathrm{~h}$ after BrdU injection, brains were fixed with $4 \%$ paraformaldehyde, and $20-\mu \mathrm{m}$ coronal sections were obtained 
with a cryostat (CM 1850, Leica). For BrdU immunostaining, brain slices were incubated in $2 \mathrm{~N} \mathrm{HCl}$ at $37^{\circ} \mathrm{C}$ for $30 \mathrm{~min}$ before incubation with mouse anti-BrdU antibody. Images of the slices were acquired with a confocal microscope (Zeiss LSM510 and Olympus FV300).

\section{Statistical Analysis}

To compare four groups, one-way ANOVA followed by the Bonferroni post hoc test for multiple comparisons was used. $\mathrm{p}$ values $<0.05$ were considered to be statistically significant.

\section{Results}

SUMOylation of DISC1-C at Lysine Residue 643

(K643) in vitro

DISC1 is found in multiple subcellular domains, and its nuclear localization and biological roles have been demonstrated [22]. This subcellular targeting may be determined in part by posttranslational modifications [25]. As a role of SUMOylation in targeting proteins to the nucleus is known [26], we hypothesized that a pool of DISC1 may be SUMOylated. We thus first predicted the SUMOylation site(s) in DISC1 by using a bioinformatics approach with the Abgent SUMOplot (http://www.abgent. $\mathrm{com} /$ sumoplot). By this method, DISC1 at lysine residue 643 (K643) was identified as a strong candidate SUMOylation site (fig. 1a). Since K643 is in the C-terminus of DISC1, we assayed in vitro SUMOylation with GSTtagged C-terminal DISC1 protein spanning amino acid residues 598-854 (DISC1-C). In the presence of ATP, which is crucial for SUMO-protein conjugation, DISC1C was SUMOylated by SUMO1, SUMO2, or SUMO3 (fig. 1b). Next, to confirm whether K643 is essential for SUMOylation in DISC1-C, we performed in vitro SUMOylation assays using mutant DISC1-C where lysine residue 643 was substituted into alanine (DISC1-CK643A). Even in the presence of ATP, DISC1-C-K643A was not SUMOylated by SUMO1 (fig. 1c). These results were consistent after normalization of the SUMOylated DISC1-C signals by the amount of the non-SUMOylated form of DISC1-C (cyan arrowhead in fig. 1c; color in online version only). Thus, these results indicate that DISC1-C is SUMOylated at lysine residue 643 in vitro.

\section{SUMOylation of DISC1 at K643 in Cells}

Next, to test whether the SUMOylation of DISC1 occurs in cells, we exogenously expressed DISC1-C together with SUMO1 in cells and examined whether DISC1-C is conjugated to SUMO. DISC1-C and SUMO1 were indeed co-immunoprecipitated, and a signal for the immu- noprecipitated protein complex was observed (red arrowhead in the IP panel in fig. $2 \mathrm{a}$; color in online version only). Of note, a signal for SUMOylation of DISC1-C was also observed in the lysates (red arrowhead in the Input panel in fig. 2a). Then, to determine whether K643 is the SUMOylation site in DISC1-C in cells, we examined whether the mutation in which lysine residue 643 was replaced with alanine (K643A) in the C-terminal of DISC1 interfered with the SUMOylation. Immunoblotting with cells transfected with DISC1-C showed a strong signal that represents SUMOylated DISC1-C, whereas such a signal was abolished in cells expressed with DISC1-CK643A (fig. 2b). These results suggest that the SUMOylation of DISC1-C occurs at lysine residue 643 in cells.

We next assessed whether the full-length DISC1 could also be SUMOylated in cells. A strong signal that represents SUMOylated DISC1 was detected when DISC1Ubc9, which enhances the SUMOylation, was exogenously expressed together with SUMO1 in cells (red arrowhead). This SUMOylation on DISC1 was abolished by co-expression of SUMO1 with DISC1-Ubc9 (C93S), a dominant negative form of Ubc9 (fig. 2c).

To address the question whether K643 is the main SUMOylation site on full-length DISC1, we compared potential SUMOylation between wild-type full-length DISC1 (DISC1) and full-length DISC1-K643A. Cells were co-expressed with DISC1 or DISC1-K643A together with SUMO1 and Ubc9. DISC1 and SUMO1 were coimmunoprecipitated, and a signal for the immunoprecipitated protein complex was observed (red arrowhead in the IP panel in fig. 2d), whereas such a signal was abolished in cells expressed with DISC1-K643A. These results were consistent after normalization of the SUMOylated DISC1 signals by the amount of the non-SUMOylated form of full-length DISC1 (cyan arrowheads in fig. 2d). Of note, a signal for SUMOylation of DISC1 was also observed in the lysates (red arrowhead in the Input panel in fig. 2d). On the basis of these results, K643 is considered the main SUMOylation site on full-length DISC1.

\section{K640 SUMOylation Site in mDISC1 (Corresponding to K643 in Human DISC1) Is Crucial for Proper \\ Proliferation of Neural Progenitor Cells in the Developing Cortex}

As DISC1 is reportedly located in multiple subcellular domains $[11,23,24]$, we tested the possible influence of SUMOylation on the subcellular distribution of DISC1. We observed decreased nuclear targeting of DISC1K643A compared to wild-type DISC1, suggesting that SUMOylation at K643 in human DISC1 (corresponding 


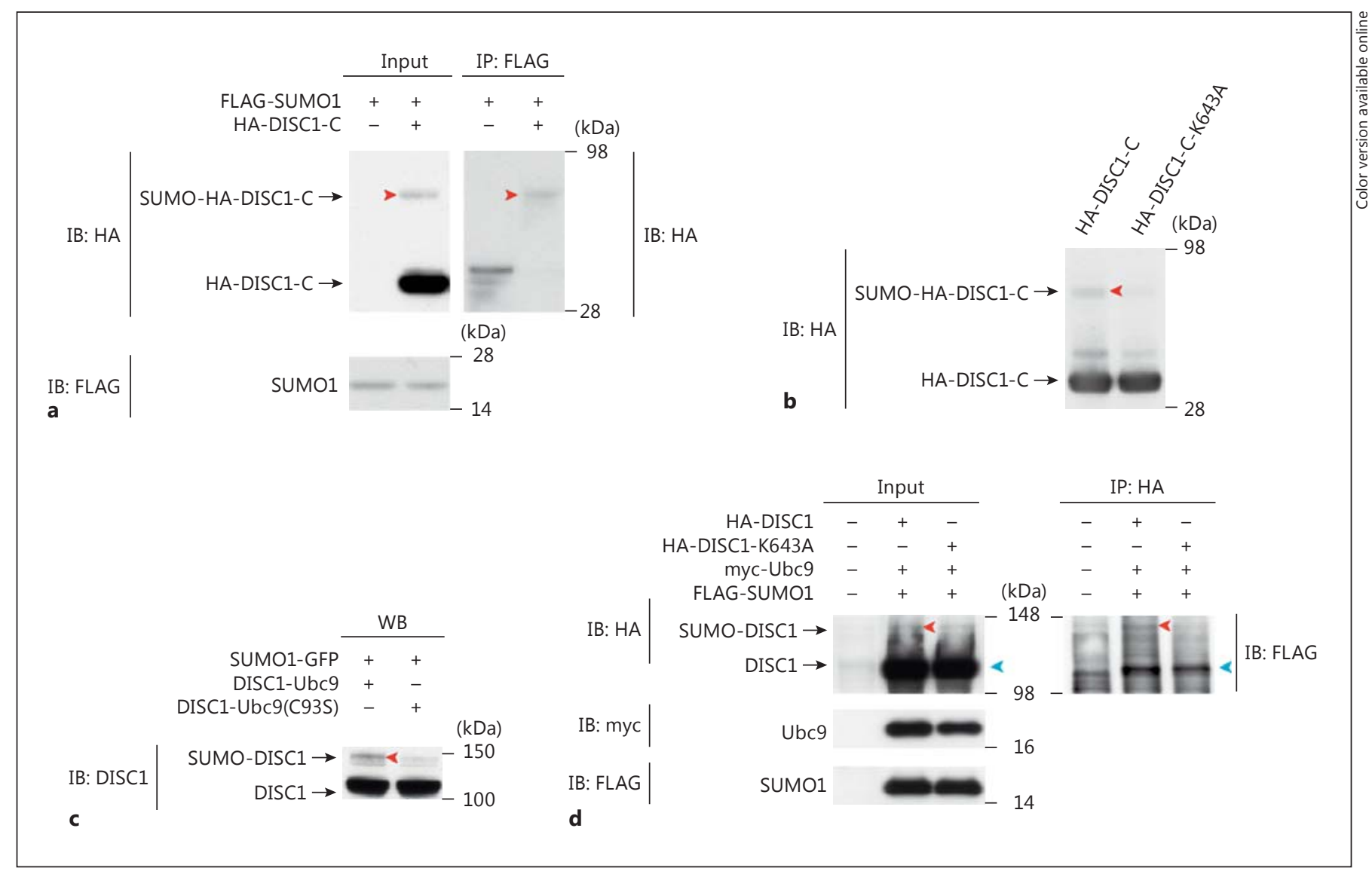

Fig. 2. SUMOylation of DISC1 at lysine residue 643 (K643) in cells. a Conjugation of HA-DISC1-C with FLAG-SUMO1 (SUMOylation of DISC1) was shown in COS7 cells. COS7 cells were transfected with HA-DISC1-C and/or FLAG-SUMO1. Cell lysates were immunoprecipitated (IP) with anti-FLAG antibody, and the immunoprecipitates were blotted with anti-HA antibody. Red arrowheads indicate SUMOylated forms of DISC1-C. b COS7 cells were transfected with HA-DISC1-C or HA-DISC1-C-K643A. Cells with HA-DISC1-C showed a strong signal, which represents a SUMOylated form of DISC1-C, suggesting SUMOylation of DISC1 with the endogenous SUMO protein. This signal was significantly abolished in cells transfected with HA-DISC1-C-K643A. The red arrowhead indicates SUMOylated forms of DISC1-C. c SUMOylation of full-length DISC1 was shown in HEK293 cells

to K640 in mDISC1) may play a role in the nuclear localization of DISC1 (online suppl. fig. 1; for all online suppl. material, see www.karger.com/doi/10.1159/000444257).

Previous studies have shown that DISC1 modulates neural progenitor cell (NPC) proliferation in vivo [25, 39]. Thus, we tested how the K640 SUMOylation site in mDISC1 affected the proliferation of NPCs by in utero gene transfer combined with BrdU pulse-labeling. Consistent with our previous study [25], the proportion of by UFDS assay. SUMO1-GFP fusion was co-transfected with DISC1-Ubc9, an E2 SUMO-conjugating enzyme fusion, or DISC1Ubc9 (C93S), a dominant negative form of Ubc9 fusion, in HEK293 cells. Cell lysates were immunoblotted (IB) with anti-DISC1 antibody. The red arrowhead indicates SUMOylated forms of DISC1. d COS7 cells were co-transfected with HA-DISC1 or HA-DISC1K643A together with FLAG-SUMO1 and myc-Ubc9. Cell lysates were immunoprecipitated with anti-HA antibody, and the immunoprecipitates were blotted with anti-FLAG antibody. Signals for SUMOylated full-length DISC1 were observed in cells transfected with HA-DISC1 (red arrowheads), whereas almost no signals were found of the same size in cells transfected with HA-DISC1-K643A. Colors refer to the online version only.

cells incorporating BrdU was significantly decreased in brains with mDISC1 knockdown (mDISC1 RNAi) compared to controls, indicating that NPC proliferation was impaired. These knockdown effects were ameliorated by co-expression with wild-type mDISC1 but not by mDISC1-K640A (fig. 3), suggesting that the K640 SUMOylation site in mDISC1 is crucial for the proper proliferation of NPCs in the developing cortex. 
Fig. 3. K640 SUMOylation site in mDISC1, corresponding to K643 in human DISC1, is crucial for proper proliferation of NPCs in vivo. Images show E15 brains that were electroporated with GFP together with control shRNA, mDISC1 shRNA, mDISC1 shRNA + wild-type (wt) mDISC1, or mDISC1 shRNA + mDISC1-K640A at E13. BrdU was injected $2 \mathrm{~h}$ before brain extraction. The proportion of BrdU-incorporating cells in the ventricular (VZ) and multipolar cell accumulation zone (MAZ) [46] in brains with mDISC1 shRNA was significantly lower than that in brains with control shRNA. This phenotype was rescued by wt mDISC1 but not by mDISC1-K640A. The graph shows the proportion of GFP/ BrdU double-positive cells to total GFPpositive cells in the VZ/MAZ. Scale bar = $20 \mu \mathrm{m}$. Error bars indicate SEM. * $\mathrm{p}<0.05$.

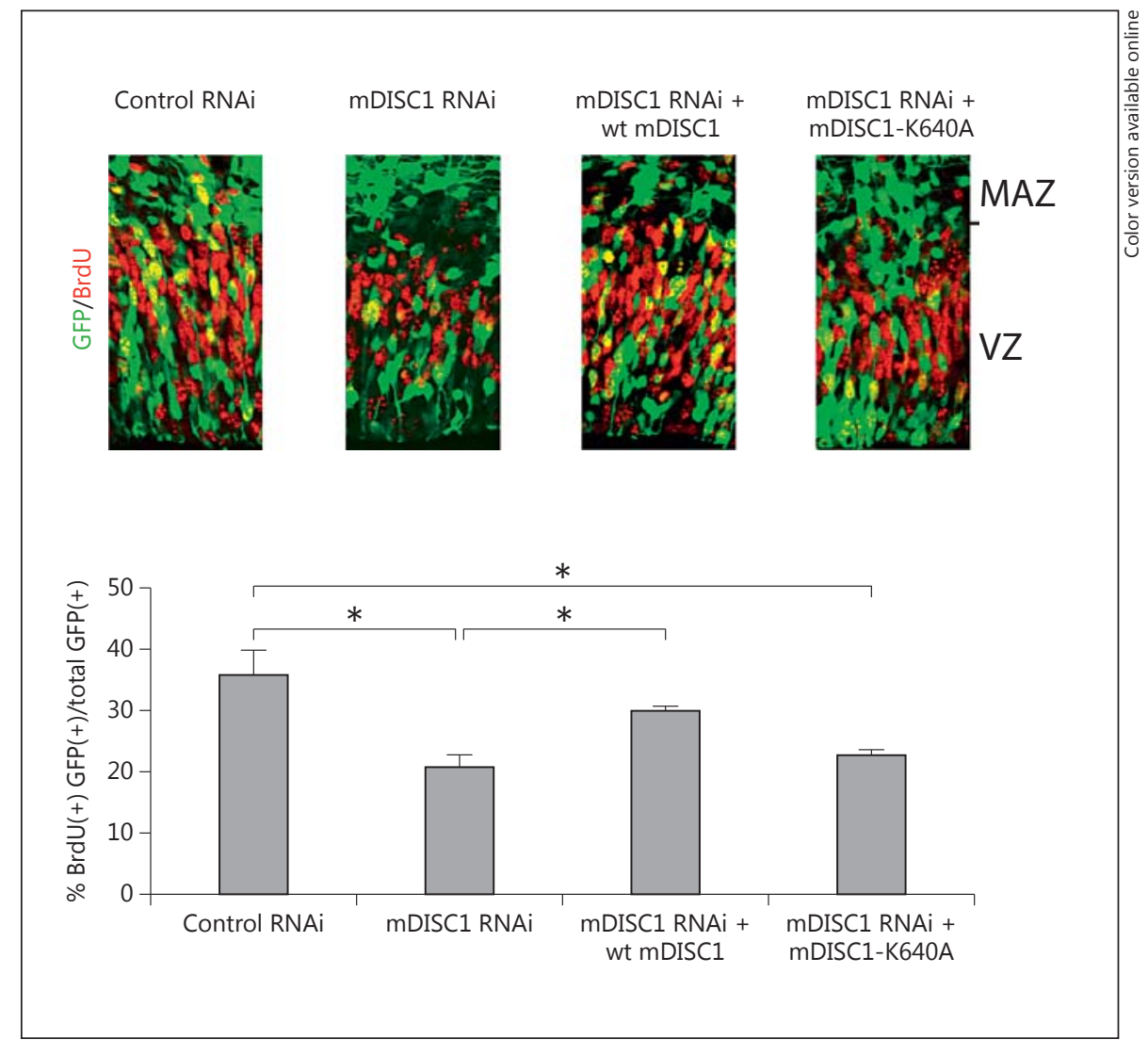

\section{Discussion}

Here, we report that DISC1 protein is SUMOylated. By combining in silico, biochemical, and cell-biological approaches, we have determined that lysine 643 in human DISC1 (which corresponds to lysine 640 in mDISC1) is a major SUMOylation site. We showed that a mutation in this residue leads to cellular deficits, including improper neural progenitor proliferation in the developing cortex.

In our biochemical analysis, the levels of SUMOylation at DISC1-K643 were relatively modest. It is known that, in general, a small pool of SUMOylated protein can have significant biological effects [40]. Therefore, the small population of SUMOylated DISC1 might have important biological effects. In cell cultures, we observed that the mutation at thelysine residue for the critical SUMOylation affected nuclear localization of DISC1. Thus far, several papers have indicated a role for nuclear DISC1 $[25,39]$. By examining autopsied brains from patients with neuropsychiatric conditions, Sawamura et al. [41] suggested that nuclear DISC1 in the orbitofrontal cortex may be involved in substance abuse. Furukubo-Tokunaga and co- workers [42] reported that DISC1 is involved in the regulation of sleep homeostasis, in particular in association with nuclear DISC1. More recently, Tsai and coworkers [43] have demonstrated that nuclear DISC1 is crucial for the regulation of phosphodiesterase-cyclic AMP signaling that underlies multiple psychiatric conditions. Taken together, although each study is still independent, without a cohesive or integrative story of nuclear DISC1 function as yet, assessing nuclear DISC1 will be important in studying the pathological mechanisms for mental illnesses. We hope that SUMOylation at K643 in human DISC1 may be a solid molecular probe to lead off such mechanistic studies.

In addition, we observed that the replacement of lysine with alanine at a critical SUMOylation site (mouse K640, which corresponds to human K643) disturbed neural progenitor proliferation in the developing cortex. In the experiments, we used in utero electroporation with DISC1-RNAi and expression constructs. The knockdown effect by the DISC1-RNAi construct used in the current study has been fully validated by multiple groups $[11,24$, $25,39]$. Furthermore, DISC1 knockdown-mediated aber- 
rant neural progenitor proliferation has been shown with multiple RNAi constructs targeting different regions of DISC1 and has been rescued by co-expressing the fulllength isoform of DISC1 in the present and in previous studies $[25,39]$. Although the significant influence of $\mathrm{mDISC1}-\mathrm{K} 640$ is not a direct demonstration that SUMOylation of DISC1 is crucial for the neurodevelopmental process, we believe this is very suggestive evidence. To address this question in a more direct manner, although it is beyond the scope of the present study, we may need to develop anti-SUMOylation-specific DISC1 antibodies. Equivalent antibodies have been frequently used as tools to study protein phosphorylation [44] and have indeed been applied to a study on DISC1 in the past [25]. There is thus a successful precedence for using anti-SUMOylation-specific antibodies in biomedical studies $[7,45]$. Such posttranslational modification-specific antibodies may become useful probes to study the pathology of mental illness.

\section{Acknowledgements}

We thank Ms. Y. Lema for preparing the figures and organizing the manuscript, and Drs. P. Talalay and N.J. Gamo for critical reading of the manuscript. This work was supported by USPHS grants MH084018 Silvio O. Conte center (A.S.), MH094268 Silvio O. Conte center (A.S. and A.K.), MH069853 (A.S.), MH085226 (A.S.), MH088753 (A.S.), MH092443 (A.S.), MH091230 (A.K.), MH96208 (K.I.), and MH105660 (A.S. and K.I.); grants from the Stanley, RUSK, S-R, and DANA foundations (A.S.); grants from NARSAD (A.S., A.K., and K.I.); grants from MSCRF (A.S. and K.I.), and the Grand Challenge Programme grants from Pfizer (G.B.).

\section{Statement of Ethics}

All animal care and use was in accordance with guidelines for the care and use of laboratory animals issued by the National Institutes of Health and The Johns Hopkins University. The authors have no ethical conflicts to disclose.

\section{Disclosure Statement}

N.J.B. is a full-time employee and a shareholder at AstraZeneca. The other authors have no conflicts of interest to report.

\section{References}

1 Samii A, Nutt JG, Ransom BR: Parkinson's disease. Lancet 2004;363:1783-1793.

2 Insel TR: Rethinking schizophrenia. Nature 2010;468:187-193.

3 Ballard C, Gauthier S, Corbett A, Brayne C, Aarsland D, Jones E: Alzheimer's disease. Lancet 2011;377:1019-1031.

4 Grundke-Iqbal I, Iqbal K, Tung YC, Quinlan M, Wisniewski HM, Binder LI: Abnormal phosphorylation of the microtubule-associated protein tau (tau) in Alzheimer cytoskeletal pathology. Proc Natl Acad Sci USA 1986;83: 4913-4917.

5 Volpicelli-Daley LA, Luk KC, Patel TP, Tanik SA, Riddle DM, Stieber A, Meaney DF, Trojanowski JQ, Lee VM: Exogenous alpha-synuclein fibrils induce Lewy body pathology leading to synaptic dysfunction and neuron death. Neuron 2011;72:57-71.

6 Grundke-Iqbal I, Iqbal K, Quinlan M, Tung YC, Zaidi MS, Wisniewski HM: Microtubuleassociated protein tau. A component of Alzheimer paired helical filaments. J Biol Chem 1986;261:6084-6089.

7 Fujiwara H, Hasegawa M, Dohmae N, Kawashima A, Masliah E, Goldberg MS, Shen J, Takio K, Iwatsubo T: $\alpha$-Synuclein is phosphorylated in synucleinopathy lesions. Nat Cell Biol 2002;4:160-164.

8 Iqbal K, Grundke-Iqbal I, Zaidi T, Merz PA Wen GY, Shaikh SS, Wisniewski HM, Alafu- zoff I, Winblad B: Defective brain microtubule assembly in Alzheimer's disease. Lancet 1986;2:421-426.

9 Haj-Yahya M, Fauvet B, Herman-Bachinsky Y, Hejjaoui M, Bavikar SN, Karthikeyan SV, Ciechanover A, Lashuel HA, Brik A: Synthetic polyubiquitinated $\alpha$-synuclein reveals important insights into the roles of the ubiquitin chain in regulating its pathophysiology. Proc Natl Acad Sci USA 2013;110:17726-17731.

10 St Clair D, Blackwood D, Muir W, Carothers A, Walker M, Spowart G, Gosden C, Evans HJ: Association within a family of a balanced autosomal translocation with major mental illness. Lancet 1990;336:13-16.

11 Hayashi-Takagi A, Takaki M, Graziane N, Seshadri S, Murdoch H, Dunlop AJ, Makino Y, Seshadri AJ, Ishizuka K, Srivastava DP, Xie Z, Baraban JM, Houslay MD, Tomoda T, Brandon NJ, Kamiya A, Yan Z, Penzes P, Sawa A: Disrupted-in-Schizophrenia 1 (DISC1) regulates spines of the glutamate synapse via Racl. Nat Neurosci 2010;13:327-332.

12 Wen Z, Nguyen HN, Guo Z, Lalli MA, Wang X, Su Y, Kim NS, Yoon KJ, Shin J, Zhang C, Makri G, Nauen D, Yu H, Guzman E, Chiang $\mathrm{CH}$, Yoritomo N, Kaibuchi K, Zou J, Christian KM, Cheng L, Ross CA, Margolis RL, Chen G, Kosik KS, Song H, Ming GL: Synaptic dysregulation in a human iPS cell model of mental disorders. Nature 2014;515:414-418.
13 Niwa M, Kamiya A, Murai R, Kubo K, Gruber AJ, Tomita K, Lu L, Tomisato S, Jaaro-Peled H, Seshadri S, Hiyama H, Huang B, Kohda K, Noda Y, O’Donnell P, Nakajima K, Sawa A, Nabeshima T: Knockdown of DISC1 by in utero gene transfer disturbs postnatal dopaminergic maturation in the frontal cortex and leads to adult behavioral deficits. Neuron 2010;65:480-489.

14 Hikida T, Jaaro-Peled H, Seshadri S, Oishi K, Hookway C, Kong S, Wu D, Xue R, Andrade M, Tankou S, Mori S, Gallagher M, Ishizuka K, Pletnikov M, Kida S, Sawa A: Dominantnegative DISC1 transgenic mice display schizophrenia-associated phenotypes detected by measures translatable to humans. Proc Natl Acad Sci USA 2007;104:14501-14506.

15 Pletnikov MV, Ayhan Y, Nikolskaia O, Xu Y, Ovanesov MV, Huang H, Mori S, Moran TH, Ross CA: Inducible expression of mutant human DISC1 in mice is associated with brain and behavioral abnormalities reminiscent of schizophrenia. Mol Psychiatry 2008;13:173$186,115$.

16 Shen S, Lang B, Nakamoto C, Zhang F, Pu J, Kuan SL, Chatzi C, He S, Mackie I, Brandon NJ, Marquis KL, Day M, Hurko O, McCaig CD, Riedel G, St Clair D: Schizophrenia-related neural and behavioral phenotypes in transgenic mice expressing truncated DISC1. J Neurosci 2008;28:10893-10904. 
17 Li W, Zhou Y, Jentsch JD, Brown RA, Tian X, Ehninger D, Hennah W, Peltonen L, Lonnqvist J, Huttunen MO, Kaprio J, Trachtenberg JT, Silva AJ, Cannon TD: Specific developmental disruption of disruptedin-schizophrenia-1 function results in schizophrenia-related phenotypes in mice. Proc Natl Acad Sci USA 2007;104:1828018285.

18 Kvajo M, McKellar H, Arguello PA, Drew LJ, Moore H, MacDermott AB, Karayiorgou M, Gogos JA: A mutation in mouse DISC1 that models a schizophrenia risk allele leads to specific alterations in neuronal architecture and cognition. Proc Natl Acad Sci USA 2008; 105:7076-7081.

19 Clapcote SJ, Lipina TV, Millar JK, Mackie S, Christie S, Ogawa F, Lerch JP, Trimble K, Uchiyama M, Sakuraba Y, Kaneda H, Shiroishi T, Houslay MD, Henkelman RM, Sled JG, Gondo Y, Porteous DJ, Roder JC: Behavioral phenotypes of Discl missense mutations in mice. Neuron 2007;54:387-402.

20 Johnson AW, Jaaro-Peled H, Shahani N, Sedlak TW, Zoubovsky S, Burruss D, Emiliani F, Sawa A, Gallagher M: Cognitive and motivational deficits together with prefrontal oxidative stress in a mouse model for neuropsychiatric illness. Proc Natl Acad Sci USA 2013; 110:12462-12467.

21 Kuroda K, Yamada S, Tanaka M, Iizuka M, Yano H, Mori D, Tsuboi D, Nishioka T, Namba T, Iizuka Y, Kubota S, Nagai T, Ibi D, Wang R, Enomoto A, Isotani-Sakakibara M, Asai N, Kimura K, Kiyonari H, Abe T, Mizoguchi A, Sokabe M, Takahashi M, Yamada K, Kaibuchi $\mathrm{K}$ : Behavioral alterations associated with targeted disruption of exons 2 and 3 of the DISC1 gene in the mouse. Hum Mol Genet 2011;20:4666-4683.

22 Brandon NJ, Sawa A: Linking neurodevelopmental and synaptic theories of mental illness through DISC1. Nat Rev Neurosci 2011;12: 707-722.

23 Camargo LM, Collura V, Rain JC, Mizuguchi K, Hermjakob H, Kerrien S, Bonnert TP, Whiting PJ, Brandon NJ: Disrupted in Schizophrenia 1 Interactome: evidence for the close connectivity of risk genes and a potential synaptic basis for schizophrenia. Mol Psychiatry 2007;12:74-86.

24 Kamiya A, Kubo K, Tomoda T, Takaki M, Youn R, Ozeki Y, Sawamura N, Park U, Kudo C, Okawa M, Ross CA, Hatten ME, Nakajima $\mathrm{K}$, Sawa A: A schizophrenia-associated mutation of DISC1 perturbs cerebral cortex development. Nat Cell Biol 2005;7:1167-1178.

25 Ishizuka K, Kamiya A, Oh EC, Kanki H, Seshadri S, Robinson JF, Murdoch H, Dunlop AJ, Kubo K, Furukori K, Huang B, Zeledon M, Hayashi-Takagi A, Okano H, Nakajima K,
Houslay MD, Katsanis N, Sawa A: DISC1-dependent switch from progenitor proliferation to migration in the developing cortex. Nature 2011;473:92-96.

26 Johnson ES: Protein modification by SUMO. Annu Rev Biochem 2004;73:355-382.

27 Muller S, Hoege C, Pyrowolakis G, Jentsch S: SUMO, ubiquitin's mysterious cousin. Nat Rev Mol Cell Biol 2001;2:202-210.

28 Mao Y, Sun M, Desai SD, Liu LF: SUMO-1 conjugation to topoisomerase I: a possible repair response to topoisomerase-mediated DNA damage. Proc Natl Acad Sci USA 2000; 97:4046-4051.

29 Bassi C, Ho J, Srikumar T, Dowling RJ, Gorrini C, Miller SJ, Mak TW, Neel BG, Raught B, Stambolic V: Nuclear PTEN controls DNA repair and sensitivity to genotoxic stress. Science 2013;341:395-399.

30 Yan Q, Gong L, Deng M, Zhang L, Sun S, Liu J, Ma H, Yuan D, Chen PC, Hu X, Liu J, Qin J, Xiao L, Huang XQ, Zhang J, Li DW: SUMOylation activates the transcriptional activity of Pax-6, an important transcription factor for eye and brain development. Proc Natl Acad Sci USA 2010;107:21034-21039.

31 Mahajan R, Delphin C, Guan T, Gerace L, Melchior F: A small ubiquitin-related polypeptide involved in targeting RanGAP1 to nuclear pore complex protein RanBP2. Cell 1997;88:97-107.

32 Jakobs A, Koehnke J, Himstedt F, Funk M, Korn B, Gaestel M, Niedenthal R: Ubc9 fusion-directed SUMOylation (UFDS): a method to analyze function of protein SUMOylation. Nat Methods 2007;4:245-250.

33 Kamiya A, Tan PL, Kubo K, Engelhard C, Ishizuka K, Kubo A, Tsukita S, Pulver AE, Nakajima K, Cascella NG, Katsanis N, Sawa A: Recruitment of PCM1 to the centrosome by the cooperative action of DISC1 and BBS4: a candidate for psychiatric illnesses. Arch Gen Psychiatry 2008;65:996-1006.

34 Ozeki Y, Tomoda T, Kleiderlein J, Kamiya A, Bord L, Fujii K, Okawa M, Yamada N, Hatten ME, Snyder SH, Ross CA, Sawa A: Disruptedin-Schizophrenia-1 (DISC-1): mutant truncation prevents binding to NudE-like (NUDEL) and inhibits neurite outgrowth. Proc Natl Acad Sci USA 2003;100:289-294.

35 Ogawa F, Kasai M, Akiyama T: A functional link between Disrupted-In-Schizophrenia 1 and the eukaryotic translation initiation factor 3. Biochem Biophys Res Commun 2005; 338:771-776.

36 Eykelenboom JE, Briggs GJ, Bradshaw NJ, Soares DC, Ogawa F, Christie S, Malavasi EL, Makedonopoulou P, Mackie S, Malloy MP, Wear MA, Blackburn EA, Bramham J, McIntosh AM, Blackwood DH, Muir WJ, Porteous DJ, Millar JK: A t(1;11) translocation linked to schizophrenia and affective disorders gives rise to aberrant chimeric DISC1 transcripts that encode structurally altered, deleterious mitochondrial proteins. Hum Mol Genet 2012;21:3374-3386.

37 Ogawa F, Malavasi EL, Crummie DK, Eykelenboom JE, Soares DC, Mackie S, Porteous DJ, Millar JK: DISC1 complexes with TRAK1 and Mirol to modulate anterograde axonal mitochondrial trafficking. Hum $\mathrm{Mol}$ Genet 2014;23:906-919.

38 Tabata H, Nakajima K: Efficient in utero gene transfer system to the developing mouse brain using electroporation: visualization of neuronal migration in the developing cortex. Neuroscience 2001; 103:865-872.

39 Mao Y, Ge X, Frank CL, Madison JM, Koehler AN, Doud MK, Tassa C, Berry EM, Soda T, Singh KK, Biechele T, Petryshen TL, Moon RT, Haggarty SJ, Tsai LH: Disrupted in schizophrenia 1 regulates neuronal progenitor proliferation via modulation of GSK $3 \beta / \beta$ catenin signaling. Cell 2009;136:1017-1031.

40 Geiss-Friedlander R, Melchior F: Concepts in SUMOylation: a decade on. Nat Rev Mol Cell Biol 2007;8:947-956.

41 Sawamura N, Sawamura-Yamamoto T, Ozeki Y, Ross CA, Sawa A: A form of DISC1 enriched in nucleus: altered subcellular distribution in orbitofrontal cortex in psychosis and substance/alcohol abuse. Proc Natl Acad Sci USA 2005;102:1187-1192.

42 Sawamura N, Ando T, Maruyama Y, Fujimuro M, Mochizuki H, Honjo K, Shimoda M, Toda H, Sawamura-Yamamoto T, Makuch LA, Hayashi A, Ishizuka K, Cascella NG, Kamiya A, Ishida N, Tomoda T, Hai T, Furukubo-Tokunaga K, Sawa A: Nuclear DISC1 regulates CRE-mediated gene transcription and sleep homeostasis in the fruit fly. Mol Psychiatry 2008;13:1138-1148, 1069.

43 Soda T, Frank C, Ishizuka K, Baccarella A, Park YU, Flood Z, Park SK, Sawa A, Tsai LH: DISC1-ATF4 transcriptional repression complex: dual regulation of the CAMP-PDE4 cascade by DISC1. Mol Psychiatry 2013;18:898908.

44 Xie Z, Sanada K, Samuels BA, Shih H, Tsai LH: Serine 732 phosphorylation of FAK by Cdk5 is important for microtubule organization, nuclear movement, and neuronal migration. Cell 2003;114:469-482.

45 Takahashi-Fujigasaki J, Arai K, Funata N, Fujigasaki H: SUMOylation substrates in neuronal intranuclear inclusion disease. Neuropathol Appl Neurobiol 2006;32:92-100.

46 Tabata H, Kanatani S, Nakajima K: Differences of migratory behavior between direct progeny of apical progenitors and basal progenitors in the developing cerebral cortex. Cereb Cortex 2009;19:2092-2105. 